\title{
Demonstration of Applying the Evaporative Cooling Technology to the 800W Transmitter
}

\author{
Cao rui ${ }^{1, a}$, Ruan Lin ${ }^{* 2, b}$ \\ ${ }^{1}$ Institute of Electrical Engineering,Chinese Academy of Science,China \\ ${ }^{1}$ Institute of Electrical Engineering,Chinese Academy of Science,China \\ acr304@mail.iee.ac.cn, ${ }^{\text {b}}$ rosaline@mail.iee.ac.cn, ${ }^{*}$ corresponding author
}

Keywords: power amplifier unit; evaporative cooling system; reliability

\begin{abstract}
In order to dissipate the heat effectively and improve the performance of $800 \mathrm{~W}$ Transmitter, we designed a set of self-circulating evaporative cooling system for the 800W power amplifier unit and made an demonstration unit. Through this application, we removed the air-cooling fin and reduced the unit's weight greatly. This system not only break the thermal bottleneck of air cooling, but also has a better cooling effect, low noise, simple structure and high reliability, besides, this application shows great cooling capability potential.
\end{abstract}

\section{Introduction}

In recent years, the design of power amplifier unit is integrated highly and air cooling mode left much to be desired, so the cooling has become bottlenecks to the transmitter's design and development. The most commonly used air-cooled technology has almost gradually reached the limit of its heat dissipation. Currently the most popular is the water cooling technology, but it also has many weaknesses: more system equipments, complex structure, and the risk of short circuit caused by cooling water leakage ${ }^{[1 \sim 4]}$. In addition, there also has some hot and advance researches: heat pipe, semiconductor refrigeration mode and so on, but these researches are not widely used now because the process is complicated and the mechanism is still being studied $\mathrm{d}^{[\sim 6]}$. To take away the heat of the surface of amplifier components and improve the reliability of the transmitter, we have to find an efficient and reliable cooling method which also has simple structure to the best.

The evaporative cooling technology takes advantage of phase change to realize the cooling effect of the object. Its cooling capability is far larger than the other heat transfer method using specific heat. This cooling technology has been successfully used to large electrical machines and electronic equipments in china. The recent application of this technology to the large electrical machine is the world famous Three Gorges hydropower project. It's the largest hydropower plant in the world. There are two sets of 840MVA hydrogenerators using this kind of evaporative cooling technology. The characteristic of this cooling technology include: high efficiency for the heat transfer, high safety and most important low energy cost or zero energy cost from the cooling system itself. All of these characteristics show cases it will be very suitable for the electronic components such as transmitter etc.

\section{Design of the evaporative cooling system}

In view of this situation, we proposed using adherent self-circulating evaporative cooling structure to cool the transmitter. There are 8 power amplifier tubes in the $800 \mathrm{~W}$ power amplifier unit, the total power usage of the $800 \mathrm{~W}$ unit is $3000 \mathrm{~W}$, the total thermal dissipation power is $2200 \mathrm{~W}$ and the thermal power of one single power amplifier tube is up to $275 \mathrm{~W}$.

1. Assemblage of liquid box and power amplifier unit

The key point of adherent self-circulating evaporative cooling system is the design of evaporative cooling liquid box. According to the different structure of heating source, we can

* corresponding author 
change the shape and adjust the layout of liquid box. The structure scheme of 800W power amplifier unit is shown in Fig.1.
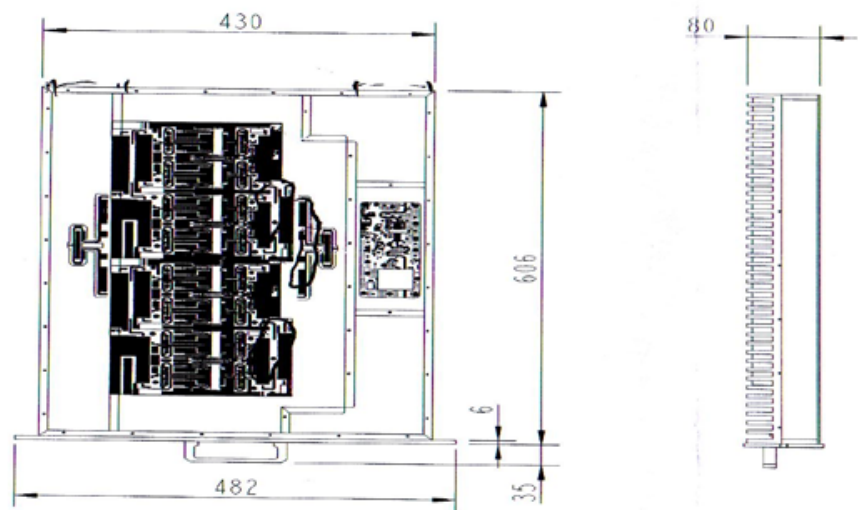

Fig.1 The structure size chart of $800 \mathrm{~W}$ power amplifier unit

When the power amplifer unit uses the air cooling method,the PCB is fixed tightly on the metal substrate which is fixed on the air cooling fin plate tightly.This metal substrate not only can transfer the heat from the power amplifer tube to the air cooling fin ,but also act as a support to fix the PCB. By using the evaporative cooling system, we removed the fin structure, the PCB is fixed tightly on the metal substrate which is fixed on the baseboard of unit tightly, and the liquid box is fixed on the back of baseboard which is slotted.The three dimensional structure diagram of 800W power amplifier unit is shown in Fig.2. We design the assemblage of liquid box and unit according to the distribution of heat source of $800 \mathrm{~W}$ power amplifier unit:There are two liquid boxes in the unit. The chart of assemblage of liquid box and unit is shown in Fig.3.

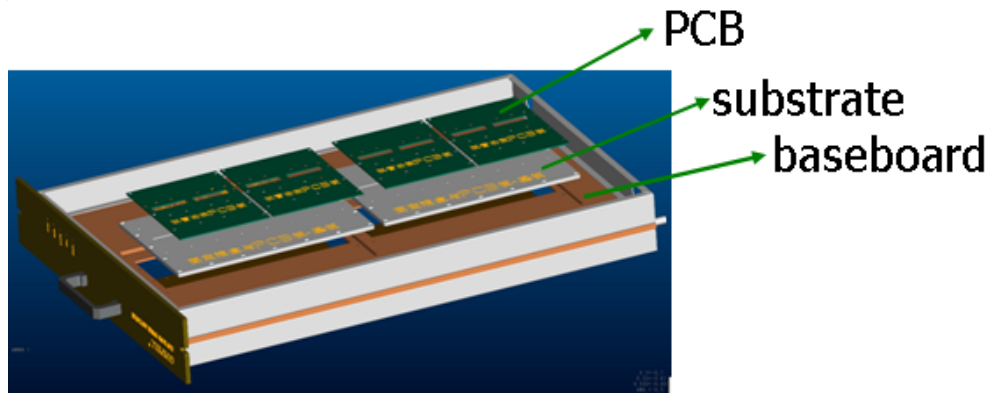

Fig.2 The three dimensional structure diagram of unit

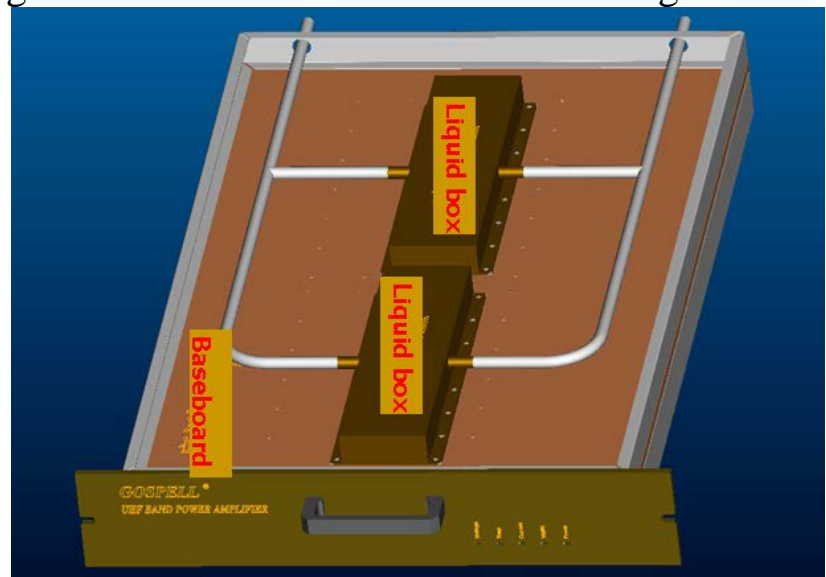

Fig.3 The chart of assemblage of liquid box and unit

2. Verification of the technological design

We use the air cooling condenser in this cooling system as the second cooling part. The capacity of air cooler depends on the total heat of transmitter.We can hang the condenser on the wall of outdoor when the transmitter is placed in the chamber.The sketch map of circulation system is shown in Fig.4. Because the 800W power amplifier unit is in development stage, we use the 200W power amplifier unit to build an experiment platform to verify the cooling effect and structure feasibility.The radio frequency function of unit was canceled,so the total heat is up to 670W. The 
experimental platform components include two 200W power amplifier units,one liquid box which is matched the structure of two units, one condenser and circulation line.The platform is shown in Fig.5.
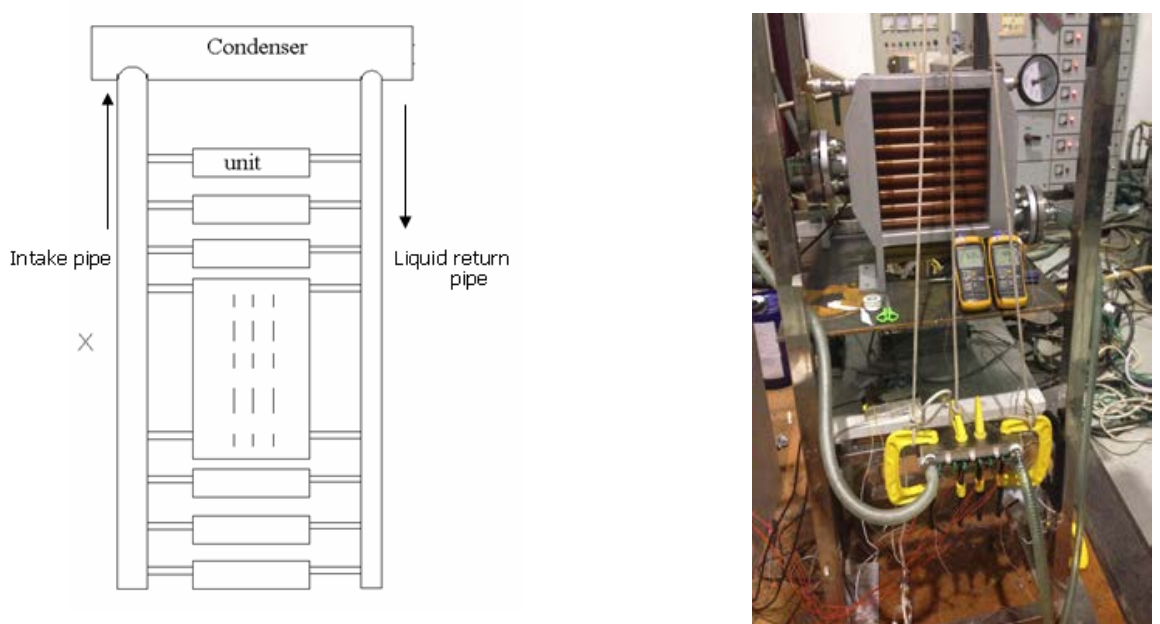

Fig.4 The sketch map of circulation system Fig.5 The experimental platform

We use 2 kinds of cooling medium(ZXB-1and ZXB-2) and 2 materials of liquid box(AandB),so there are 4 kinds of working condition,and we need to monitor the power amplifier tube, the substrate and the baseboard's temperature in the experiment. When the temperature of the environment is $27.1^{\circ} \mathrm{C}$,we can get the results as shown in Tab.1.

Tab.1 The temperature of components of the unit

\begin{tabular}{|c|c|c|c|c|c|c|}
\hline Liquidbox & \multicolumn{3}{|c|}{ ZXB-1 } & \multicolumn{3}{c|}{ ZXB-2 } \\
\hline A & $\mathrm{T}_{\mathrm{t}}: 78^{\circ} \mathrm{C}$ & $\mathrm{T}_{\mathrm{s}}: 65^{\circ} \mathrm{C}$ & $\mathrm{T}_{\mathrm{b}}: 56^{\circ} \mathrm{C}$ & $\mathrm{T}_{\mathrm{t}}: 72^{\circ} \mathrm{C}$ & $\mathrm{T}_{\mathrm{s}}: 60^{\circ} \mathrm{C}$ & $\mathrm{T}_{\mathrm{b}}: 49^{\circ} \mathrm{C}$ \\
\hline B & $\mathrm{T}_{\mathrm{t}}: 73^{\circ} \mathrm{C}$ & $\mathrm{T}_{\mathrm{s}}: 59^{\circ} \mathrm{C}$ & $\mathrm{T}_{\mathrm{b}}: 48^{\circ} \mathrm{C}$ & $\mathrm{T}_{\mathrm{t}}: 67^{\circ} \mathrm{C}$ & $\mathrm{T}_{\mathrm{s}}: 55^{\circ} \mathrm{C}$ & $\mathrm{T}_{\mathrm{b}}: 43^{\circ} \mathrm{C}$ \\
\hline
\end{tabular}

The temperature of the surface of power amplifier tube is required to keep below $80^{\circ} \mathrm{C}$,so we can take this temperature to the boundary condition in the simulation, in addition,the material of the liquid box is set to B,and the property of medium is set to ZXB-1.By the simulation we can get the distribution of the temperature of baseboard and substrate which is shown in Fig.6.

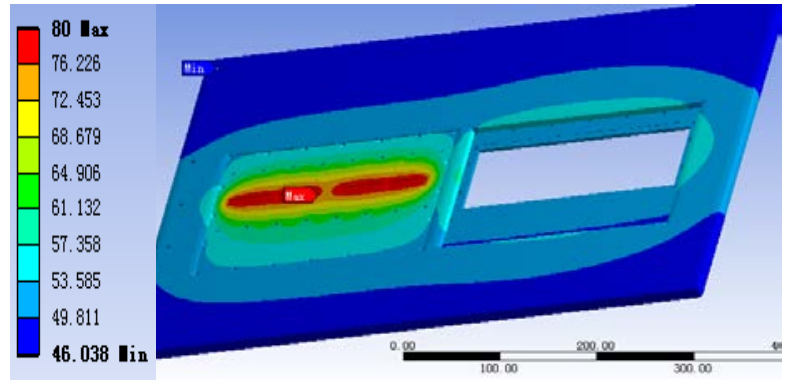

Fig.6 The temperature of baseboard and substrate

From the simulation,we can get the results :1.The temperature of the surface of power amplifier tube is kept below $80^{\circ} \mathrm{C}$;2. The temperature of the surface of substrte which is near the liquid box is about $60^{\circ} \mathrm{C} ; 3$. The temperature of the surface of substrte which is far away from the liquid box is about $50^{\circ} \mathrm{C}$. The experimental results and the simulation results are consistent which is proving that the heat capacity of liquid box can meet the cooling demand of power amplifier unit.

\section{Conclusion}

In this paper,we design a set of self-circulating evaporative cooling system for the $800 \mathrm{~W}$ power amplifier unit.Through the experiment and simulating calculation,we find that the cooling capacity 
of this set of cooling system can fully meet the need for the cooling of power amplifier unit. So comparing with air cooling and water cooling, this system not only break the thermal bottleneck of air cooling, but also has a better cooling effect, low noise, simple structure and high reliability, besides, this application shows great cooling capability potential.

\section{References}

[1] Chen Deng Ke.New technologies of electronics cooling [J].Chinese Journal of Low Temperature Physics,2005,27(3):255-262

[2] Pastukhov V.G.,Maidanik Yu F.,Vershinin C.V.Miniature loop heat pipes for electronics cooling[J].Applied Thermal Engineering.2003,23(9):1125-1135

[3] Cooper G,Herskovits E.A Bayesian Metod for the Induction of Probabilistic Networks from Data[J].Machine Learning,1992,9(4):309-347

[4] Chen Haisheng,Yang Wei,He Yurong, etal.Heat transfer and flow behaviour of aqueous suspensions of titanate nano-tubes(nanofluids) [J].Powder Techn,2008,183:63

[5] Leonard L1Vasiliev1Heat pipes in modern heat exchangers

[6] Applied Thermal Engineering, 2005 (25): 18-20 